\title{
Antibiotics prescribed to febrile under-five children outpatients in urban public health services in Burkina Faso
}

\author{
Léon G. Blaise Savadogo ${ }^{1,2^{\star}}$, Bernard Ilboudo ${ }^{1}$, Maurice Kinda ${ }^{1}$, Nacro Boubacar $^{3}$, \\ Philippe Hennart ${ }^{2}$, Michèle Dramaix², Philippe Donnen ${ }^{2}$

\footnotetext{
${ }^{1}$ Department of Epidemiology and Public Health, Child and Mother Health, Nutrition and Survival Unit, Institut Supérieur des Sciences de la Santé (IN.S.SA) (Bobo Dioulasso University), Bobo-Dioulasso, Burkina Faso;

*Corresponding Author: gueswende@hotmail.com

${ }^{2}$ CR2-Epidemiology-Biostatistics-Clinical Research, School of Public Health, Université Libre de Bruxelles (ULB), Brussels, Belgium

${ }^{3}$ Bobo-Dioulasso Pediatric Hospital and University of Ouagadougou, Ouagadougou, Burkina Faso
}

Received 15 November 2013; revised 19 December 2013; accepted 5 January 2014

Copyright (C) 2014 Léon G. Blaise Savadogo et al. This is an open access article distributed under the Creative Commons Attribution License, which permits unrestricted use, distribution, and reproduction in any medium, provided the original work is properly cited. In accordance of the Creative Commons Attribution License all Copyrights (c) 2014 are reserved for SCIRP and the owner of the intellectual property Léon G. Blaise Savadogo et al. All Copyright (c) 2014 are guarded by law and by SCIRP as a guardian.

\section{ABSTRACT}

Appropriate use of antibiotics remains critical for success in achieving MDG4. The aim of this study was to investigate antibiotics prescribing practices to febrile under-five children outpatients in urban public health services in a low income country. Methods: From March to April 2013, a cross-sectional epidemiological study of care facilities visit by under-five age, for febrile illness, was carried out in urban health services in Bobo-Dioulasso, Burkina Faso. Patient demographics, diagnoses and medications were recorded. We calculated for each diagnoses several indicators for antibiotics use. Results: Our study showed an over-prescription of antibiotics at the university teaching hospital $(\mathbf{7 8 . 0 8 \%})$ and at the first level facilities $(57.71 \%)$ for under-five outpatients for febrile illness. There was evidence of high antibiotic prescription in children with diarrhea (more than 9 on 10 at university teaching hospital of diarrhea cases and $60 \%$ at the first level facilities), in children with Upper respiratory tract infections (respectively $60 \%$ and $85.2 \%$ of cases at university teaching hospital and at the first level facilities) and in children with malaria (respectively $47.5 \%$ and $17.6 \%$ of cases at university teaching hospital and at the first level facilities). Overuse, misuse and inappropriately prescribed antibiotic coexisted in our results: at university teaching hospital $\mathbf{9 0 . 9 \%}$ of diarrhea cases, $60 \%$ of URTI cases,
$47.5 \%$ of malaria cases received antibiotic prescription; at first level heath care facilities $85.2 \%$ of URTI, $17.6 \%$ of malaria cases received an prescribed antibiotic and $\mathbf{1 1 . 8 \%}$ of LRTI did not received a prescribed antibiotic. Developing countries have poor access to newer antibiotics and irrational antibiotics use remains a global problem. Overuse and misuse of antibiotics combat, rigorous infectious diseases diagnosis, antimicrobial resistance consequences education of users and health professional's prescribers, and improved surveillance of antimicrobial resistance, must be strengthened.

\section{KEYWORDS}

Antibiotics; Children; Febrile; Outpatients; Public Health Services

\section{INTRODUCTION}

The 2012 report of levels and trends in child mortality, showed global substantial progress made towards achieving Millennium Development Goal 4 (MDG 4). The number of under-five deaths worldwide has declined from nearly 12 million in 1990 to 7 million in 2011 [1]. But in Sub-Saharan Africa, under-five age children mortality rate is still the highest and most of countries will not achieve MDG 4 by 2015. In those countries, the leading causes of deaths among under-five age children are pneumonia, diarrhea, malaria and undernutrition.

Reducing under-five mortality rate in low income 
countries depends on effective interventions to prevent and to treat infectious diseases. Appropriate use of antibiotics remains critical for success in achieving MDG4. Antibiotics have increased life expectancy [2]. In Africa, when hospitalization of sick infants is unfeasible, outpatient therapy with antibiotics is an effective option [3]. For treatment of patients with antibiotics for community-acquired pneumonia in ambulatory settings, amoxicillin is an alternative to co-trimoxazole [4]. But, inappropriate use of antibiotics continues to be a health major problem in developing countries and little is known about paediatric prescribing practices. Common problems of irrational use of antibiotics include polypharmacy, overuse, failure to prescribe in accordance with clinical guidelines and inappropriate self-medication [5]. In developing countries, low percent of patients in the public and private sectors are treated according to clinical guidelines; many children with acute diarrhea don't receive necessary oral rehydration therapy and most of them receive unnecessary antibiotics; a large percent of children with viral upper respiratory tract infection receive antibiotics inappropriately [6]. Consequences include antimicrobial resistance, adverse drug reactions and medication errors, lost resources (patients and countries) and eroded patient confidence to the health facilities. Much has been done in the past 25 years to improve the use of antibiotics worldwide. But at present, investment in promoting rational use of antibiotics, appears to be relatively little, in low income counties [7]. Irrational antibiotics use can lead to a public health crisis and improve situation is a major challenge for countries. Evidence for continuing irrational use of antibiotics has to be reported to promote monitoring of medicines use and implementation of interventions for medicines rational use. The aim of this study was to investigate antibiotics prescribing practices to febrile under-five children outpatients in urban public health services in a low income country.

\section{METHODS}

\subsection{Study Population, Design and Site}

From March to April 2013, a cross-sectional epidemiological study of care facilities visit by under-five age, for febrile illness, was carried out in first-line urban public health centers and in university teaching hospital in Bobo-Dioulasso, Burkina Faso. The health system is organized in two urban districts. Each district has around 12 first-line urban public care facilities. The second big university teaching hospital of Burkina Faso is in BoboDioulasso. We selected in each urban health district, one first-line public health center and the university teaching hospital for the study. The study concerned under-five age children in a health seeking process, for febrile ill- ness, at selected health facilities. During the study period, each day, parent consent under five febrile children, in an outpatient health seeking process, was enrolled consecutively.

\subsection{Study Sample Size}

From the two first-line public health centers, 376 children have been enrolled and 153 from the university teaching hospital.

\subsection{Data Collection}

Each day, patient demographics, diagnoses and medications were recorded by trained care providers, noninvolved to the consultation. Health seeking process and demographic were directly recorded from the patient interview. The outcomes of the consultation: symptoms, clinical examination, diagnose, medications were recorded from the clinical document after the end of the consultation. In resource-poor countries, diagnosis is based on identifying clinical syndromes from international guidelines, as microbiological investigations are restricted. In this study we considered consultation outcome diagnoses without analyzing misdiagnosis as prescribing have been done on this basis. From international guidelines, lower respiratory tract infection (LRTI) was defined as pneumonia or any other type of LRTI. Cough with fast breathing, lower chest wall indrawing, fever, coarse crackles or bronchial breath sounds or dullness to percussion, grunting, were in favour of pneumonia (severe or non-severe) or other lower respiratory tract infection. Upper respiratory tract infections (URTI) was defined as presence of cough, nasal discharge, mouth breathing and fever without general danger signs or signs of pneumonia. Acute (watery) diarrhea was defined as more than three loose stools per day and no blood in stools. Malaria (severe or not) based on the story, symptoms (change of behaviour, confusion, drowsiness, altered consciousness and generalized weakness), clinical assessment with special attention to level of consciousness, rate and depth of respiration and pallor. For malaria diagnosis, microscopy for parasitaemia was feasible at the university teaching hospital and rapid diagnostic test is diagnostic at first-lines health care centers. Neonatal infection symptoms and signs can be non-specific, the signs include: not feeding well, convulsions, drowsy or unconscious, movement only when stimulated or no movement at all, fast breathing, grunting, severe chest indrawing.

\subsection{Statistical Analyses}

The data were analyzed using SPSS software version 20. Usual statistics methods were applied to estimate statistics parameters. 
To describe antibiotics prescription in febrile illness of under five children, we calculated, in each health care level (university teaching hospital and first-line health care facilities), several indicators for global antibiotics use: \% patients with an prescribed antibiotic; \% patients with an prescribed antibiotic without antimalarial; \% patients with an prescribed antibiotic with an antimalarial; \% patients with an prescribed antimalarial without an antibiotic; \% patients with an prescribed medicine without an antibiotic or an antimalarial.

We calculated for each diagnoses, in each health care level, \% patients with a prescribed antibiotic. Accent has been made on neonatal infection, lower respiratory tract infection, upper respiratory tract infections, diarrhea and malaria.

\section{RESULTS}

Age median (Min-Max) was 6.80 (0 - 55.92) months among encounters at university teaching and 16.54 (0 60) months among the first-level facilities patients. Table 1 describes patients demographic in each health care level (Table 1).

Before attending the university teaching hospital, $30.1 \%$ of health seeking febrile children received medicine and from them, 47.7\% received antibiotic (Amoxicilline, Cotrimoxazole, Ceftriaxone, Gentamycine).

Before attending the first level facilities, 59.3\% of health seeking febrile children received medicine and from them, 2.3\% received antibiotic (Penicilline, Amoxicilline, Cotrimoxazole).

Main diagnoses were neonatal infection, malaria, lower respiratory tract infection (LRTI), diarrhea, upper respiratory tract infections (URTI) and combination of those diseases at the university teaching hospital (Figure 1) and were malaria, lower respiratory tract infection (LRTI), diarrhea, upper respiratory tract infections (URTI) and combination of those diseases at the health care first-level (Figure 2). The Other diseases regroup urinary tract infection, otitis, mastoiditis, septic arthritis, osteomyelitis, asthma, undernutrition, heart failure, renal failure and sickle cell.

Table 1. Patients demographic at each health care level.

\begin{tabular}{ccc}
\hline & $\begin{array}{c}\text { University teaching hospital } \\
\mathrm{n}=153\end{array}$ & $\begin{array}{c}\text { First-level facilities } \\
\mathrm{n}=376\end{array}$ \\
\hline Age (months) & & \\
0 - 6 mo (\%) & 47.7 & 17.5 \\
7 - 12 mo (\%) & 17.7 & 19.7 \\
13 - 30 mo (\%) & 24.8 & 35.1 \\
31 - 60 mo (\%) & 9.8 & 27.7 \\
Sex & & \\
Boys (\%) & 56.9 & 56.9 \\
Girls (\%) & 43.1 & 43.1 \\
\hline
\end{tabular}

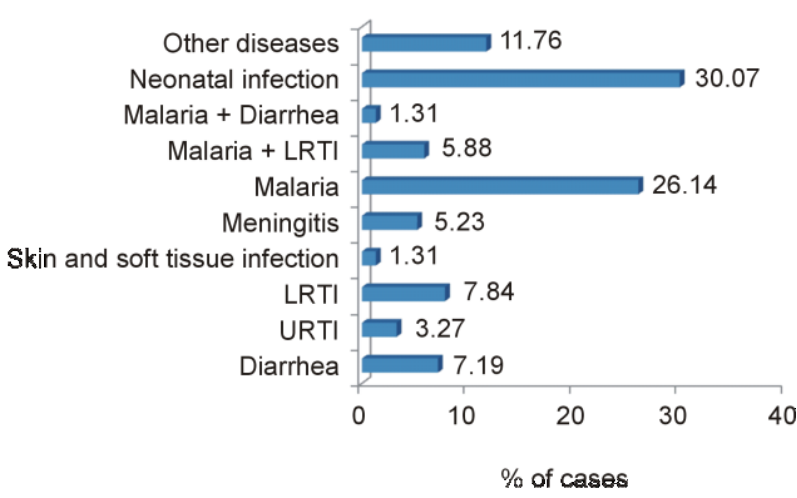

Figure 1. Diagnoses at the university teaching hospital.

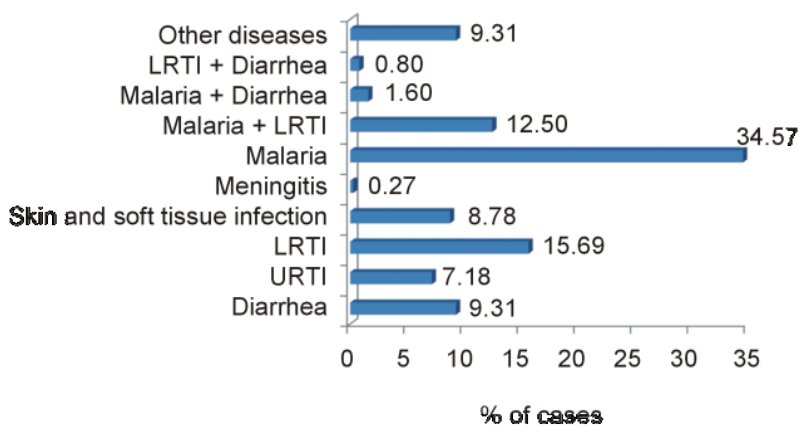

Figure 2. Diagnoses at first-level health care facilities.

At the university teaching hospital, \% patients with a prescribed antibiotic were $78.08 \%$ and $57.71 \%$ at the first level facilities (Figure 3).

Figures 4 and 5 give overview of \% of cases with prescribed antibiotic by diagnose in each health care level.

Prescribing for diarrhea: At university teaching hospital, more than 9 on 10 (90.9\%) of diarrhea cases received antibiotic prescription (54.5\% antibiotic alone, and $36.4 \%$ antibiotic associated to an antimalarial). Only 1 on 10 cases received anti-diarrheal without antibiotic. No diarrhea cases received oral rehydration therapy (ORT). All diarrhea associated to malaria cases received a prescribed antibiotic. At the first level facilities, $60 \%$ of diarrhea cases received antibiotic prescription; no cases received ORT and when associated to malaria, all cases received a prescribed antibiotic.

Prescribing for Lower respiratory tract infection (LRTI): At university teaching hospital, more than 9 on $10(91.7 \%)$ of LRTI cases received antibiotic prescription; 8.3\% did not received an antibiotic. When associated to malaria, all the cases received a prescribed antibiotic. At the first level facilities, 7 on 8 of LRTI cases (88.2\%) received antibiotic prescription; $11.8 \%$ did not receive a prescribed antibiotic. When associated to malaria or diarrhea, respectively, $97.9 \%$ and 100 of cases received an antibiotic prescription. 


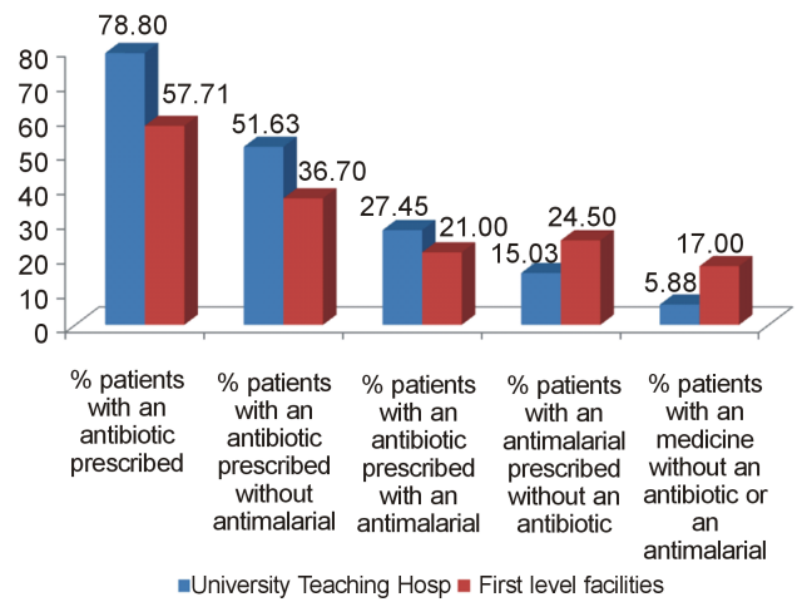

Figure 3. \% patients with an antibiotic prescribed.

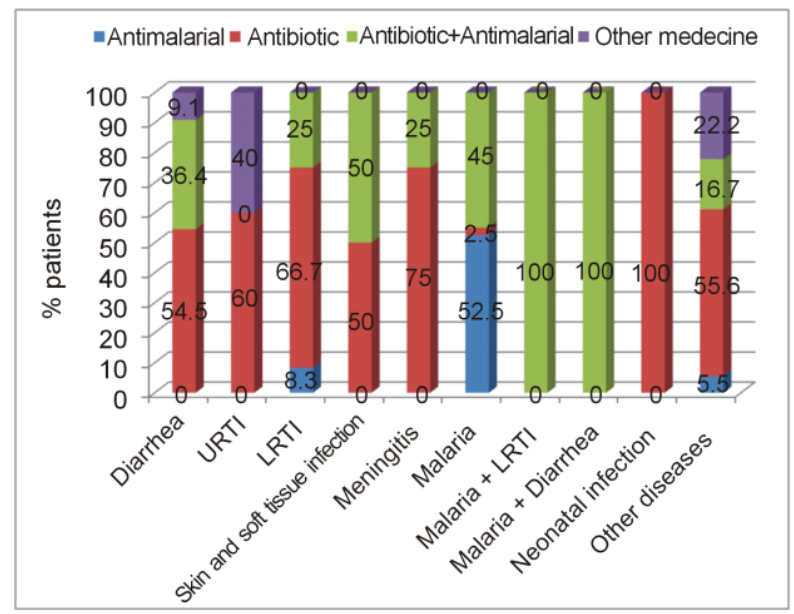

Figure 4. \% of cases (patients) with prescribed antibiotic by diagnose at university teaching hospital.

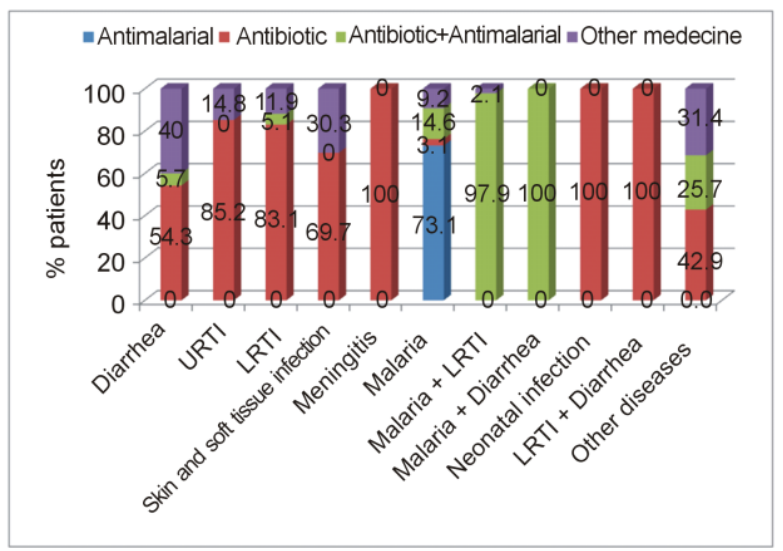

Figure 5. \% of cases (patients) with prescribed antibiotic by diagnose at the first level facilities.

Prescribing for Upper respiratory tract infections (URTI): at university teaching hospital and at the first level facilities, respectively, $60 \%$ and $85.2 \%$ of URTI cases, received a prescribed antibiotic.
Prescribing for Skin and soft tissue infection: At university teaching hospital and at the first level facilities, respectively, $100 \%$ and $69.7 \%$ of cases, received a prescribed antibiotic.

Prescribing for Malaria: At university teaching hospital and at the first level facilities, respectively, $47.5 \%$ (2.5\% antibiotic without an antimalarial) and $17.6 \%$ (3.1\% antibiotic without an antimalarial) of cases received a prescribed antibiotic.

Prescribing for Meningitis: at the two health care levels, all meningitis cases and all neonatal infections cases, received a prescribed antibiotic.

At university teaching hospital and at the first level facilities, respectively, $72.3 \%$ and $68.6 \%$ of others diseases cases received an prescribed antibiotic (Other diseases regroup urinary tract infection, otitis, mastoiditis, septic arthritis, osteomyelitis, asthma, under nutrition, heart failure, renal failure and sickle cell).

\section{DISCUSSION}

Our study showed an over-prescription of antibiotics at the university teaching hospital (78.08\%) and at the first level facilities (57.71\%) for under-five outpatients for febrile illness. We did not differentiate between appropriate and inappropriate antibiotic prescribing, so irrational use of antibiotic may be more extremely serious problem in our context. A summarize of studies reported between 1990 and 2006, about medicines use in primary care in developing and transitional countries, showed that percentage of patients with an antibiotic prescribed remained stable over time at between $40 \%$ and $50 \%$. From the same studies, only half of the patients or less were prescribed medicines according to clinical guidelines [6]. In our study, there was evidence of high antibiotic prescription in children with diarrhea (more than 9 on 10 at university teaching hospital of diarrhea cases received antibiotic prescription and $60 \%$ at the first level facilities) in children with Upper respiratory tract infections (URTI) (respectively $60 \%$ and $85.2 \%$ of URTI cases received an prescribed antibiotic at university teaching hospital and at the first level facilities) and in children with malaria (respectively $47.5 \%$ and $17.6 \%$ of malaria cases received an prescribed antibiotic at university teaching hospital and at the first level facilities). In our study, there was no compliance with standard treatment guidelines for acute diarrhoea; the use of antibiotics for acute diarrhoea was high from university teaching hospital and from first level health care facilities and there was no ORT prescribing. In a rational prescribing in pediatrics in a low income country, authors showed an overprescription of antibiotics in children with cough and coryzal symptoms (54.5\%) and simple diarrhoea (44.8\%) [8]. There is little evidence of antibiotics efficiency on 
diarrhoea treatment in young children in low and middle income countries [9]. There is evidence that antibiotic use and growing resistance [10]. Resistance is becoming major public health problem. Antibiotics multi-resistance has been documented in several countries and is associated with increased lethality. In developing countries, where overall burden of infectious diseases is higher, the most serious resistance problems are associated with Gram-negative bacteria and tuberculosis and may result in increased risk of death [11]. Authors reported evidence of correlation between outpatient antibiotic use and penicillin-resistant pneumococci, emphasizing the importance of restrictive antibiotic prescribing policies [12]. Overuse, misuse and inappropriately prescribed antibiotic coexisted in our results: at university teaching hospital $90.9 \%$ of diarrhea cases, $60 \%$ of URTI cases, $47.5 \%$ of malaria cases received antibiotic prescription; at first level heath care facilities $85.2 \%$ of URTI, $17.6 \%$ of malaria cases received an prescribed antibiotic and $11.8 \%$ of LRTI did not received a prescribed antibiotic. From summarize of studies during 2004-2006, the percentage of children under 5 years old with reported viral URTI who were treated with antibiotics reached over 70; over $30 \%$ of children less than 5 years old with reported pneumonia were not treated with appropriate antibiotics and only $70 \%$ of pneumonia cases receive an appropriate antibiotic [6]. If antibiotics become ineffective, increased morbidity and mortality rate will be observed.

This study has been done in a short period (two months) and in only during the dry season. More long observational study that covers the two seasons will give better view of antibiotics use in health's facilities in Burkina Faso.

\section{CONCLUSIONS}

Appropriate treatment of acute diarrhoea with greater use of oral rehydration solution and less use of antibiotics; greater use of appropriate antibiotics for pneumonia and less inappropriate use of antibiotics for upper respiratory tract infection, are urgently need.

Medicine use is rational (appropriate, proper, correct) when patients receive the appropriate medicines, in doses that meet their own individual requirements, for an adequate period of time, and at the lowest cost both to them and the community. Irrational (inappropriate, improper, incorrect) use of medicines is when one or more of these conditions are not met.

Developing countries have poor access to newer antibiotics and irrational antibiotics use remains a global problem. Despite of this situation, basic policies needed to ensure appropriate use of antibiotics are not implemented in most of those countries. Overuse and misuse of antibiotics combat, rigorous infectious diseases diagnosis, antimicrobial resistance consequences education of users and health professional's prescribers, and improved surveillance of antimicrobial resistance, must be strengthened.

\section{ACKNOWLEDGEMENTS}

Commission Universitaire pour le Développement (CUD), Belgium, founded research assistants for field data collecting.

\section{REFERENCES}

[1] You, D., New, J.R. and Wardlaw, T. (2012) The report 2012 of levels \& trends in child mortality. Unicef, WHO, The World Bank.

http://www.unicef.org/videoaudio/PDFs/UNICEF_2012 child_mortality_for_web_0904.pdf

[2] Zarb, P. and Goossens, H. (2012) Human use of antimicrobial agents. Revue Scientifique et Technique, 31, 121133.

[3] Zaidi, A.K., Tikmani, S.S., Warraich, H.J., Darmstadt, G.L., Bhutta, Z.A., Sultana, S. and Thaver, D. (2012) Community-based treatment of serious bacterial infections in newborns and young infants: A randomized controlled trial assessing three antibiotic regimens. Pediatric Infectious Disease Journal, 31, 667-672.

http://dx.doi.org/10.1097/INF.0b013e318256f86c

[4] Lodha, R., Kabra, S.K. and Pandey, R.M. (2013) Antibiotics for community-acquired pneumonia in children. Cochrane Database of Systematic Reviews, 6, Article ID: CD004874.

http://dx.doi.org/10.1002/14651858.CD004874.pub4

[5] Holloway, K. and Van Dijk, L. (2011) The World Medicines Situation 2011, rational use of medicines. 3rd Edition, World Health Organization, Geneva.

[6] WHO (2009) Medicines use in primary care in developing and transitional countries: Fact book summarizing results from studies reported between 1990 and 2006. World Health Organization, Geneva.

http://www.who.int/medicines/publications/primary_care 8April09.pdf

[7] WHO (2006) Using indicators to measure country pharmaceutical situations: Fact book on WHO Level I and Level II monitoring indicators. World Health Organization, Geneva.

http://apps.who.int/medicinedocs/index/assoc/s14101e/s1 4101e.pdf

[8] Risk, R., Naismith, H., Burnett, A., Moore, S.E., Cham, M. and Unger, S. (2013) Rational prescribing in paediatrics in a resource-limited setting. Archives of Disease in Childhood, 98, 503-509.

http://dx.doi.org/10.1136/archdischild-2012-302987

[9] Abba, K., Sinfield, R., Hart, C.A. and Garner, P. (2009) Antimicrobial drugs for persistent diarrhoea of unknown or non-specific cause in children under six in low and middle income countries: Systematic review of randomized controlled trials. BMC Infectious Diseases, 9, 24. 
[10] Ganguly, N.K., Arora, N.K., Chandy, S.J., Fairoze, M.N., Gill, J.P., Gupta, U., Hossain, S., Joglekar, S., Joshi, P.C., Kakkar, M., Kotwani, A., Rattan, A., Sudarshan, H., Thomas, K., Wattal, C., Easton, A., Laxminarayan, R. and Global Antibiotic Resistance Partnership (GARP)—India Working Group (2011) Rationalizing antibiotic use to limit antibiotic resistance in India. The Indian Journal of
Medical Research, 134, 281-294.

[11] Blomberg, B. (2008) Antimicrobial resistance in developing countries. Tidsskr Nor Laegeforen, 128, 2462-2466.

[12] Goossens, H. (2009) Antibiotic consumption and link to resistance. Clinical Microbiology and Infection, 15, S12S15. http://dx.doi.org/10.1111/j.1469-0691.2009.02725.x

\section{ABBREVIATIONS}

MDG: Millennium Development Goal

LRTI: Lower Respiratory Tract Infection

URTI: Upper Respiratory Tract Infections 